Classification

Physics Abstracts

$86.10 \mathrm{~K}$

\title{
Capteur climatique
}

\author{
R. Pelletret
}

ENSIMEV, 59326 Le Mont Houy, Valenciennes, France

\author{
et $\mathrm{X}$. Berger \\ CNRS Groupe d'Ecothermique Solaire, Centre de Recherches Bâtiments Solaires, \\ BP 21, 06562 Valbonne Cedex, France
}

(Reçu le 17 septembre 1982, révisé le 7 décembre, accepté le 15 décembre 1982)

\begin{abstract}
Résumé. - L'association système héliothermique-pompe à chaleur constitue une des voies les plus prometteuses pour réduire la consommation de chauffage en énergie primaire des bâtiments. Le couplage d'une pompe à chaleur eau/eau à un capteur sans vitrage à ruissellement d'eau froide présente de par sa simplicité un intérêt technicoéconomique évident. Les apports calorifiques proviennent non seulement de l'énergie solaire incidente mais aussi de l'enthalpie de l'air. De nombreuses expériences ont permis d'affiner les modẻlisations et de constater l'insuffisance des gains par condensation par temps couvert. La circulation de solutions hygroscopiques corrige cette faiblesse. Les mesures effectuées avec de telles solutions laissent entrevoir de larges possibilités, en particulier celle d'un stockage intersaisonnier par gradient de concentration.
\end{abstract}

\begin{abstract}
The association solar collectors and heatpumps is one of the most ready promiser way to reduce the primary energy consumption for heating of building. Coupling a water to water heatpump with a streaming coldmater unglazed solar collector presents, of course its simplicity, a real technical and economical interest. The calorific supplies proceed not only from solar energy but also from air enthalpy. Modelizations have been refined ihrough numerous experiments and the insufficiency of condensation supply has been showed when the sky is clouded. The streaming of hygroscopic liquids corrects this weak point. The achieved measures with such liquid let us believe to a large development in particulary for long time heat storage by variation of concentration degree.
\end{abstract}

Introduction. - Le chauffage solaire des habitations se heurte à la difficulté majeure d'une mauvaise correspondance temporelle entre besoins et apports.

Cettę constatation conduit à adjoindre un stockage au système de captage et même un chauffage d'appoint pour organiser valablement une installation. Une étude sur les stratégies de chauffage (Réf. [1]) a permis d'aboutir à la conclusion suivante : un système de captage qui, par beau temps, capterait $20 \%$ de moins d'énergie qu'un système classique mais qui, par mauvais temps, capterait $50 \%$ de ce qu'il capte par beau temps (alors que le système classique ne capte pratiquement rien) permettrait de couvrir en région Niçoise $15 \%$ de plus de besoins en chauffage que le système classique, et plus de $45 \%$ en plus en région Parisienne.

Récupérer de l'énergie climatique par temps couvert c'est fonctionner avec un fluide à très basse température afin de capter non seulement le rayonnement solaire incident, mais aussi l'enthalpie de l'air ambiant. C'est le propre du capteur climatique. Celui-ci présente diverses configurations :

- écoulement d'eau ou de fluides hygroscopiques en surface (capteur surfacique à ruissellement),

- écoulement d'air au voisinage d'un matériau hygroscopique (capteur volumique),

- écoulement d'eau ou de fréon dans un radiateur.

Outre les problèmes technologiques de mise au point, l'étude du capteur climatique rencontre ceux des phénomènes évaporatifs et convectifs qui s'avèrent complexes (mouvements de matière, tension superficielle...). Par ailleurs les solutions hygroscopiques présentent l'intérêt de constituer très simplement par variation de leur concentration, des stockages indépendants de la température.

L'article qui suit ne fait que présenter les premières expérimentations concernant ce type de capteur. $\mathrm{Si}$ 
l'on songe que la vapeur d'eau est le principal régulateur thermique de notre atmosphère, on se convainc que les études sur les phénomènes évapo-convectifs et sur les systèmes associés n'en sont qu'à leur début.

1. Les équations du bilan de fonctionnement. - La forme la plus simple pour un capteur climatique consiste en un ruissellement d'eau sur un plan incliné.

Si l'on considère la lame d'eau (ou de liquide hygroscopique) qui constitue la partie active du capteur, un certain nombre de phénomènes d'échanges thermiques agissent simultanément sur elle; certains ont des actions opposées comme le rayonnement du ciel et rayonnement solaire; d'autres comme l'évaporation et la convection sont inséparables l'un de l'autre car ils mettent en jeu des mouvements de matière.

1.1 LeS APPORTS SOLAIRES. - a) Une partie du rayonnement solaire est réfléchie à la surface du dioptre eau-air, le taux $\rho$ de réflexion étant déterminé par la loi de Fresnel :

$$
\rho=\frac{1}{2}\left[\frac{\sin ^{2}\left(\theta_{2}-\theta_{1}\right)}{\sin ^{2}\left(\theta_{2}+\theta_{1}\right)}-\frac{\operatorname{tg}^{2}\left(\theta_{2}-\theta_{1}\right)}{\operatorname{tg}^{2}\left(\theta_{2}+\theta_{1}\right)}\right],
$$

où $\theta_{1}, \theta_{2}$ sont les angles d'incidences et de réfraction, liés par la relation de Descartes.

En tenant compte de la répartition rayonnement direct-rayonnement diffus $(24 \%$ de rayonnement diffus par ciel clair), le taux de réflexion du rayonnement global est voisin de $3 \%$ par ciel clair pour les incidences solaires inférieures à $40^{\circ}$. b) Le taux d'absorption a du rayonnement pendant la traversée de la lame d'eau est donnée par une loi exponentielle du type :

$$
a=1-\mathrm{e}^{-k x},
$$

avec $: k:$ coefficient d'absorption,

$$
x \text { : épaisseur de la lame d'eau. }
$$

En pratique l'épaisseur $x$ est telle que $a$ est inférieur à $1 \%$.

c) Une partie du rayonnement transmis, estimée à $8 \%$ (par suite de la qualité de la surface sombre), est réfléchie sur la surface qui constitue le capteur et en presque totalité ressort. Le rayonnement absorbé échauffe le support de la lame d'eau et l'énergie est restituée au fluide par conduction-convection (il existe un phénomène inertiel de constante de temps 2 à 4 minutes). La valeur du coefficient global d'absorption du rayonnement solaire par l'eau a été finalement résumée dans le tableau $I$.

Tableau I. - Absorption du rayonnement solaire en

\begin{tabular}{|c|c|c|}
\hline & Beau temps & Ciel couvert \\
\hline $\begin{aligned} i & <45^{\circ} \\
45^{\circ}<i & <55^{\circ} \\
55^{\circ} & <i<65^{\circ} \\
65^{\circ} & <i<75^{\circ}\end{aligned}$ & $\begin{array}{l}89 \% \\
88 \% \\
86 \% \\
81 \%\end{array}$ & $86 \%$ \\
\hline
\end{tabular}
fonction de l'incidence du rayonnement direct et $d u$ type de temps.

1.2 LeS ÉCHANGeS PAR RAYONNEMENT. - La température de rayonnement du ciel est donnée par la relation (Réf. [2])

$$
T_{\text {ciel }}=\left[T_{\text {air }}+K(\text { heure })+L \text { (heure) }\left(t_{\text {rosée }}-t_{\text {air }}\right)\right]\left(0,752+0,0048 t_{\text {rosée }}\right)^{0,25}
$$

où $K$ et $L$ sont des coefficients fonctions de l'heure à partir du lever du soleil.

Tableau II. - K et L en fonction de l'heure à partir du lever du soleil.

\begin{tabular}{|c|c|c|c|c|c|c|c|c|}
\hline $\begin{array}{l}\text { Heures à compter } \\
\text { du lever du soleil }\end{array}$ & \multicolumn{1}{|c|}{0} & \multicolumn{1}{|c|}{3} & \multicolumn{1}{|c|}{6} & 9 & \multicolumn{1}{|c|}{12} & \multicolumn{1}{|c|}{15} & \multicolumn{1}{|c|}{18} & \multicolumn{1}{|c|}{21} \\
\hline$K$ & 3,86 & 0,18 & 2,90 & 4,85 & 5,65 & 6,45 & 7,18 & 7,65 \\
$L$ & 0,399 & 0,276 & 0,290 & 0,311 & 0,364 & 0,435 & 0,620 & 0,616 \\
\hline
\end{tabular}

L'énergie $W_{\mathbf{R}}$ échangée par rayonnement est la somme des énergies échangées avec le ciel et le sol, dans la proportion des angles solides de vision de chacun, et :

$$
W_{\mathrm{R}}=\frac{1+\cos i}{2} \frac{\sigma}{\frac{1}{\varepsilon_{\text {ciel }}}+\frac{1}{\varepsilon_{\text {eau }}}-1}\left(T_{\text {ciel }}^{4}-T_{\text {eau }}^{4}\right)+\frac{1-\cos i}{2} \frac{\sigma}{\frac{1}{\varepsilon_{\text {sol }}}+\frac{1}{\varepsilon_{\text {eau }}}-1}\left(T_{\text {sol }}^{4}-T_{\text {eau }}^{4}\right),
$$

avec : $i$ : inclinaison du capteur

$\sigma=5,67 \times 10^{-8}$ S.I. constante de Boltzmann. 
1. 3 LeS ÉCHANGES CONVECTIFs. - L'énergie $W_{\mathrm{C}}$ échangée par convection suit une loi du type

$$
W_{\mathrm{C}}=h_{\mathrm{c}}\left(t_{\mathrm{air}}-t_{\text {eau }}\right)
$$

avec $h_{\mathrm{c}}=6+3 \mathrm{~V}^{0,8}$ (Réf. [3]),

$V$ : vitesse du vent en $\mathrm{m} / \mathrm{s}$.

Remarque : Dans l'expérience effectuée, l'écart entre la température d'air et celle de l'eau n'était que de quelques degrés et la vitesse du vent faible, le plus souvent inférieure à $2 \mathrm{~m} / \mathrm{s}$. L'importance du choix de l'expression à adopter pour $h_{\mathrm{c}}$ restait donc faible, les incertitudes engendrées dans le calcul de $W_{\mathrm{C}}$ ne pouvant être que de quelques watts.

L'expression ci-dessus est celle adoptée au départ pour l'étude du bilan de fonctionnement. La corrélation existante entre les phénomènes évaporatif et convectif, étudiée ci-après, contraint à considérer une relation laissant reliés les deux effets.

1.4 LES ÉCHANGES PAR ÉVAPORATION-CONDENSATION. - L'étude des échanges évapo-condensatifs constitue la partie centrale de la recherche effectuée. Le motif en est double :

- le fonctionnement optimal du capteur climatique implique de tirer un profit maximal de ce mode d'échanges,

- la connaissance des lois qui régissent ces échanges énergétiques est encore très imparfaite, surtout si l'on quitte l'échelle du laboratoire.

L'évaporation-condensation, tout comme la convection, sont deux phénomènes d'échanges thermiques qui ont lieu simultanément et qui font intervenir des mouvements de matière.

Le mouvement de transport de la lame d'eau et sa finesse (épaisseur $\cong 0,1$ à $0,2 \mathrm{~mm}$ ) sont tels qu'il y a homogénéisation permanente en température (et en concentration dans le cas de l'utilisation de saumures). Par contre, l'agitation de l'air au voisinage de la lame d'eau peut être très variable (influence sur l'épaisseur des couches limites thermique et dynamique).

Une étude concernant les déperditions des piscines (Réf. [4]) a réuni une dizaine de relations établies à partir de mesures diverses, et dont l'accord entre elles est loin d'être parfait.

1.4.1 La loi de Dalton. - Une première relation sur l'évaporation-condensation est donnée par la loi de Dalton (Réf. [5]) :

- la pression d'un mélange de plusieurs gaz parfaits ne donnant lieu à aucune réaction chimique, est la somme des pressions partielles des différents constituants à la température considérée. La différence des pressions de vapeur saturante eau-air $\left(p-h p^{\prime}\right)$ entraîne un échange de matière $M$ ou d'énergie $W E$ par évaporation ou condensation suivant une relation de la forme :

$$
M_{\text {évap. }}=K f(V)\left(p-h p^{\prime}\right)
$$

avec

$h:$ hygrométrie relative en $\%$,

$V$ : vitesse d'agitation de l'air,

$p$ et $p^{\prime}$ : pressions de vapeur saturante. Elles sont des fonctions des températures d'air et d'eau.

Une interpolation par un polynôme de Lagrange a permis d'établir une formule (Réf. [6]) qui, mise sous la forme de Hörner, s'écrit :

$$
\begin{array}{r}
P_{\mathrm{s}}=0,605 \times 10^{-6}\left\{\left[\left(t_{\mathrm{s}}+7,066\right) t_{\mathrm{s}}+908,88\right] t_{\mathrm{s}}+\right. \\
+9567\} \quad(\text { en bar })
\end{array}
$$

avec : $0^{\circ}<t_{\mathrm{s}}<40^{\circ}$; erreur maximale $\leqslant 1 \%$.

En prenant pour $f(V)$ la formule de Gilles, l'expression de l'énergie $W_{\mathrm{E}}$ évaporée ou condensée s'écrit :

$$
W_{\mathrm{E}}=25 \Delta p\left(1+\frac{V}{15}\right),
$$

avec $\Delta p=p-h p^{\prime}$ en $\mathrm{mb}$.

Cette relation théorique suppose une indépendance totale entre la convection et l'évaporation-condensation et ne tient pas compte de la présence d'une couche limite.

Elle ne décrit malheureusement la réalité que dans certaines conditions d'expérience, ainsi que le montrent les mesures effectuées et présentées plus loin.

1.4.2 La loi de Fick. - La loi de Fick ou loi de diffusion s'exprime par :

$$
\dot{m}=-D_{\mathrm{c}} \frac{\partial C}{\partial x},
$$

(analogie avec les transferts de chaleur. Loi de Fourier $\left.\varphi=-\lambda \frac{\partial T}{\partial x}\right)$, avec :

$\dot{m}$ : flux massique d'un constituant du mélange $\left(\mathrm{kg} / \mathrm{m}^{2} . \mathrm{s}\right)$,

$C$ : concentration du constituant considéré dans le mélange $\left(\mathrm{kg} / \mathrm{m}^{3}\right)$,

$D_{\mathrm{c}}$ : coefficient de diffusion moléculaire $D_{\mathrm{c}}(T)=$ $=2,26 \times 10^{-5} \times \frac{1}{p} \times \frac{T}{273}$.

Cette loi exprime le fait que l'agitation moléculaire entraîne la diffusion de la matière des points de fortes concentrations aux points de faibles concentrations.

Les équations différentielles modélisant les processus de transfert de quantité de mouvement, de chaleur et de masse (Réf. [14]) (diffusion de la vapeur d'eau dans l'air) sont toutes trois de même type :

$$
u \frac{\partial A}{\partial x}+v \frac{\partial A}{\partial y}=K \frac{\partial^{2} A}{\partial y^{2}},
$$

( $u, v$, composantes normales et tangentielles de la vitesse de l'air).

La solution de l'équation de la chaleur conduit à la relation $N u=k \operatorname{Re}^{0,5} \mathrm{Pr}^{1 / 3}$. 
Par analogie le transfert massique s'exprime par une corrélation entre plusieurs nombres sans dimensions : le nombre de Sherwood Sh, de Reynolds Re et celui de Schmidt $S c$

$$
S h=K R e^{0,5} S c^{1 / 3} .
$$

De plus : $N u / S h=\left(D_{\mathrm{c}} / a\right)^{1 / 3}$ où $a$ est la diffusivité thermique de l'air $a=\lambda / \rho C p . a / D_{\mathrm{c}}$ est un nombre adimensionnel : le nombre de Lewis noté Le. Or

$$
\frac{N u}{S h}=\frac{h_{\mathrm{c}}}{h_{\mathrm{D}}} \times \frac{D_{\mathrm{c}}}{\rho C p} \times \frac{1}{a},
$$

où $h_{\mathrm{D}}$ est le coefficient d'échange par diffusion moléculaire, $h_{\mathrm{c}}$ le coefficient d'échange convectif.

D'où :

$$
h_{\mathrm{D}}=\frac{h_{\mathrm{c}}}{\rho C p}\left(\frac{1}{L e}\right)^{2 / 3} .
$$

Par ailleurs soit $h_{\mathrm{L}}$ le coefficient de transfert de chaleur par changement d'état liquide-vapeur.

$$
h_{\mathrm{D}}\left(C_{\mathrm{s}}-C_{0}\right)=\frac{h_{\mathrm{L}}}{L}\left(P_{\mathrm{vs}}-P_{\mathrm{v} 0}\right),
$$

$C_{s}$ et $C_{0}$ étant les concentrations, $L$ la chaleur latente de l'eau, or :

$$
P_{\mathrm{vs}}=C_{\mathrm{s}} r_{\mathrm{v}} T_{\mathrm{s}} \text { et } P_{\mathrm{v} 0}=C_{0} r_{\mathrm{v}} T_{0} .
$$

D'où en appliquant la loi de Clapeyron à l'air et en considérant $T_{\mathrm{m}}=\frac{T_{\mathrm{s}}+T_{0}}{2}$ on a :

$$
h_{\mathrm{L}}=\frac{L}{r_{\mathrm{v}} T_{\mathrm{m}}} \frac{h_{\mathrm{c}}}{\rho_{\mathrm{m}} C_{\mathrm{pm}}}\left(\frac{1}{L e}\right)^{2 / 3} .
$$

Soit :

$$
h_{\mathrm{L}}=\frac{L}{r_{\mathrm{v}} T_{0}} \frac{h_{\mathrm{c}}}{\rho_{0} C_{\mathrm{p}}}\left(\frac{1}{L e}\right)^{2 / 3} .
$$

D'où :

$$
h_{\mathrm{L}} \cong 1,67 h_{\mathrm{c}} \mathrm{W} / \mathrm{m}^{2} \cdot \mathrm{mb}
$$

Le transfert thermique total dépend donc de l'agitation de l'air (coefficient $h_{\mathrm{c}}$ ) et des pressions partielles de vapeur saturante :

$$
W_{\mathrm{E}}=\Delta p h_{\mathrm{L}}=1,67 \Delta p f(V) .
$$

Le coefficient de transfert par convection était $h_{\mathrm{c}}=6+3 V^{0,8}$ (Mac Adams).

Nous obtenons donc une deuxième formule dans laquelle la vitesse du vent n'intervient pas de la même façon.

Loi de Fick :

$$
W_{\mathbf{E}}=\left(10+5 V^{0,8}\right) \Delta p .
$$

1.5 APPORTS CONDUCTIFS PAR L'ABSORBEUR. - Le support de la lame d'eau peut être différemment constitué :

- s'il est un isolant épais (cas de l'expérience), les apports par la face arrière de l'absorbeur sont considérés comme négligeables,

- s'il est un matériau épais (dalle en béton, sol) un apport existe qui est fonction de la conduction du matériau,

- s'il est une plaque fine, il existe des apports convectifs, condensatifs et radiatifs comme ceux existants entre l'eau et l'air. Seul l'effet évaporatif n'existe pas (on peut admettre que l'eau condensée est éliminée par écoulement le long du plan incliné).

2. Modélisation par ordinateur. - Les équations de départ de l'étude (Réf. [8]) exprimant les divers échanges énergétiques ont été programmées sur ordinateur. Le capteur de largeur unitaire, a été divisé en tranches de $10 \mathrm{~cm}$ de long; la modélisation du comportement sur un hiver complet a été effectuée pour différentes régions avec un pas de calcul d'une heure. En région niçoise une description binaire du climat a été adoptée (conformément aux analyses décrites en Réf. [9]). Elle correspond à une année type moyenne. Pour les autres régions (44 Stations), les paramètres moyennés par la Météorologie Nationale sur les années 1951-1960 ont été utilisées.

La simulation a donné les résultats suivants :

il existe une surface maximale d'écoulement au-delà de laquelle, pour un débit donné, les pertes et les apports, en moyenne, s'équilibrent. Cette surface peut s'exprimer par une relation linéaire :

$$
S\left(\mathrm{en} \mathrm{m}^{2}\right)=k D(\mathrm{~kg} / \mathrm{s})
$$

où $k$ est un coefficient dépendant du lieu considéré, et variant fortement suivant les conditions météorologiques.

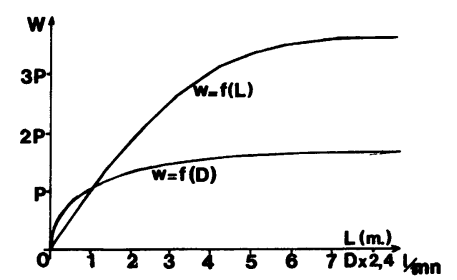

Fig. 1. - Représentation paramétrique de l'énergie $W$ récupérée à Nice (moyenne sur l'hiver) en fonction d'une part de la longueur $L$ du capteur pour un débit de $2,4 \mathrm{l} / \mathrm{mm}$, et d'autre part du débit d'eau $D$ pour une longueur de capteur de $3 \mathrm{~m}$ et un fluide en entrée à une température de $6^{\circ}$.

[Parametric representation of the collected energy in Nice (average for winter) as a function of the length of the collector for a flux of $2.41 / \mathrm{mm}$ and as a function of the water flux for a length of three meters and a temperature of liquid initially at six degrees celsius.] 
La figure 1 exprime sous forme paramétrée l'énergie récupérée en moyenne à Nice en fonction respectivement du débit d'eau et de la longueur du capteur.

- L'inclinaison optimale du capteur, étant donné la distribution des types de temps, est de $25^{\circ}$ en région niçoise (Réf. [9]), cet optimum est assez plat et varie peu d'une région à l'autre.

Cela provient de l'importance du rayonnement diffus qu'il convient de capter, surtout par mauvais temps; de même l'orientation optimale est voisine de $15^{\circ}$ Ouest du fait que la température maximale est obtenue une à deux heures après le passage au méridien. Par ailleurs, en région niçoise, l'hygrométrie est maximale en fin d'après-midi, ce qui contribue aussi à accroître les apports d'après-midi par rapport à ceux du matin (Réf. [10]).

2.1 Modélisation De FONCTIONNEMENT. RésulTATS DES SIMULATIONS. - La figure 2 indique pour le

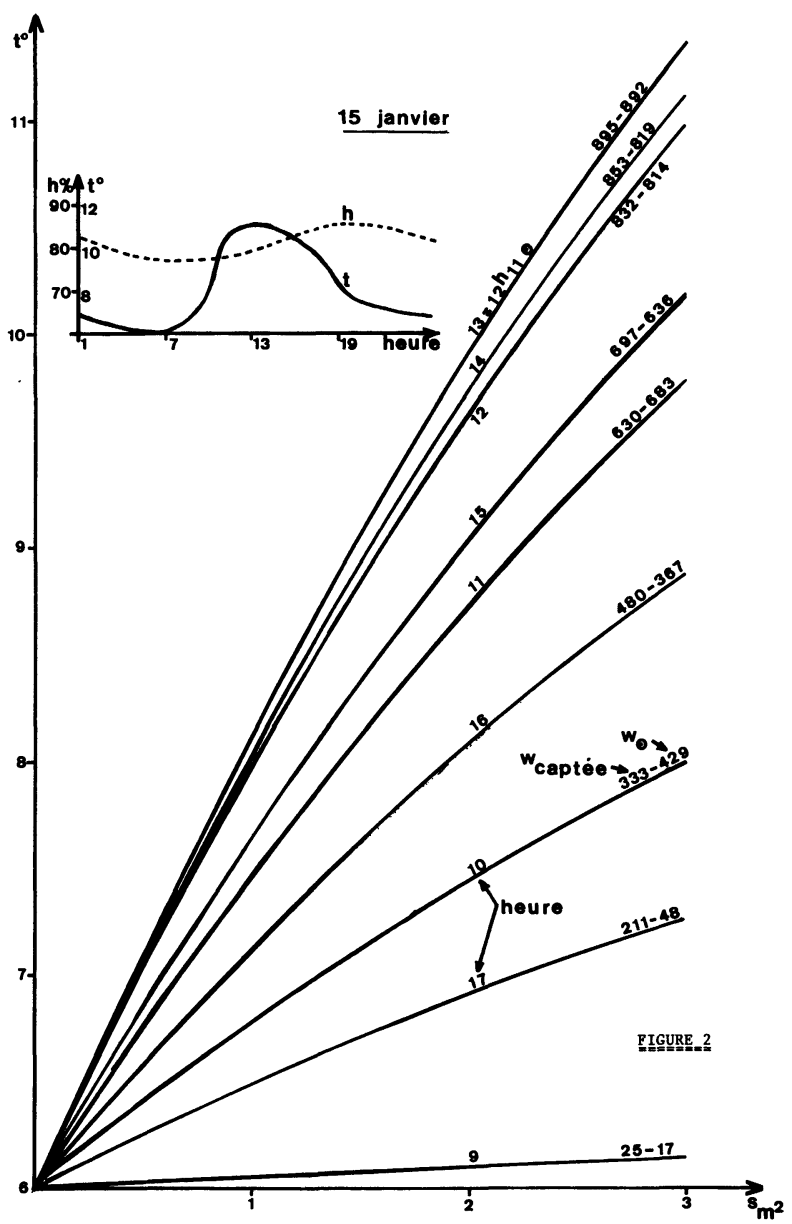

Fig. 2. - Energie captée (pour $3 \mathrm{~m}^{2}$ ) et température de sortie de l'eau en fonction de l'heure et de la surface de capteur pour un débit de $2,41 / \mathrm{mm}$ et une température d'entrée de $6^{\circ}$. En encadré hygrométrie relative et température de l'air extérieur. Nice au 15 janvier par ciel couvert.

[Collected energy (on $3 \mathrm{~m}^{2}$ ) and outler temperature of water as a function of the hour and the length of the collector (flux of $2.41 / \mathrm{mm}$ and temperature of liquid initially at $6^{\circ} \mathrm{C}$ ) (Nice, January 15 clouded sky).]
15 janvier à Nice l'énergie captée en fonction de la longueur de capteur et de l'heure solaire de la journée pour un ciel couvert. L'ensemble des résultats pour tous les mois et les deux types de temps considérés en région niçoise est donné dans le tableau III. Un certain nombre de conclusions peuvent en être tirées :

- on récupère par mauvais temps, en moyenne, $48,5 \%$ de l'énergie captée par beau temps, pour une température d'entrée de l'eau de $6^{\circ} \mathrm{C}$. Cela confirme les études antérieures (Réf. [11-12]) qui montraient que pour une température d'entrée de $4^{\circ} \mathrm{C}$ ce chiffre pouvait atteindre $60 \%$ et descendait à $40 \%$ pour une température d'entrée de $9^{\circ} \mathrm{C}$. On a donc affaire à un capteur ayant un rendement important par mauvais temps ce qui lui confère un bon amortissement et lui permet de diminuer l'importance du stockage associé.

L'ensoleillement n'est pas le seul critère mais l'enthalpie de l'air importe autant (Fig. 3). C'est pourquoi une région comme celle de $\mathrm{La}$ Rochelle conviendrait particulièrement bien pour un tel capteur :

- on récupère l'après-midi $25 \%$ de plus d'énergie que le matin; il s'ensuit qu'un tel capteur n'a pas besoin d'être associé à d'importants stockages inertiels par déphasage dans le temps puisque les calories sont captées principalement en milieu d'après-midi ; son fonctionnement n'est d'ailleurs pas limité aux seules heures d'ensoleillement,

- si l'on appelle efficacité de captage le rapport énergie récupérée sur énergie solaire incidente par jour, on obtient parfois par mauvais temps des efficacités supérieures à 1 ,

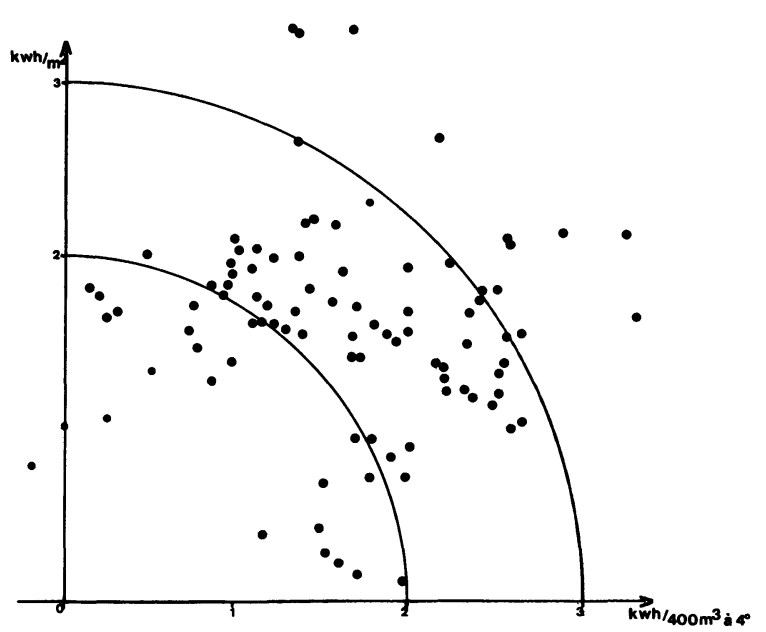

Fig. 3. - Corrélation énergie solaire incidente sur un plan horizontal - enthalpie atmosphérique (énergie récupérée par refroidissement à $4^{\circ}$ de $400 \mathrm{~m}^{3}$ d'air) (Nice - hiver 6566) Réf. [11].

[Correlation between solar energy on a horizontal surface and collected energy by air cooling of $400 \mathrm{~m}^{3}$ until $4{ }^{\circ} \mathrm{C}$ (Nice, winter 65-66) Réf. [11].] 
- pour assurer tous les besoins d'une maison $\left(V=350 \mathrm{~m}^{3}, G=1,2 \mathrm{~W} / \mathrm{m}^{2}{ }^{\circ} \mathrm{C}\right)$ pendant une journée de mauvais temps en janvier il faudrait $45 \mathrm{~m}^{2}$ de capteurs. Avec un stockage des apports excédentaires obtenus pendant les jours de beau temps ce chiffre baisserait à $30 \mathrm{~m}^{2}$. Une telle surface est tout à fait raisonnable à concevoir. Avec une stratégie d'utilisation d'appoint (Réf. [1]) elle pourrait encore descendre jusqu'à $15 \mathrm{~m}^{2}$. Le capteur à ruissellement se présente donc comme un système d'avenir d'autant que son prix est faible puisqu'il peut être constitué par une toiture ou un plan incliné goudronné (le sol fournirait alors des apports positifs). L'eau de ruissellement pourrait être stockée à $10^{\circ}$ environ dans une cuve de $10 \mathrm{~m}^{3}$ enterrée, le sol $\left(T_{\text {sol }}=13^{\circ}\right.$ à Nice $)$ fournirait $5 \%$ d'apports supplémentaires (Réf. [10]).

2.2 ProblèMe POSÉ PAR L'EFFET D'ÉVAPORATIONCONDENSATION. - Une température d'eau en dessous de la température de rosée en présence d'apports
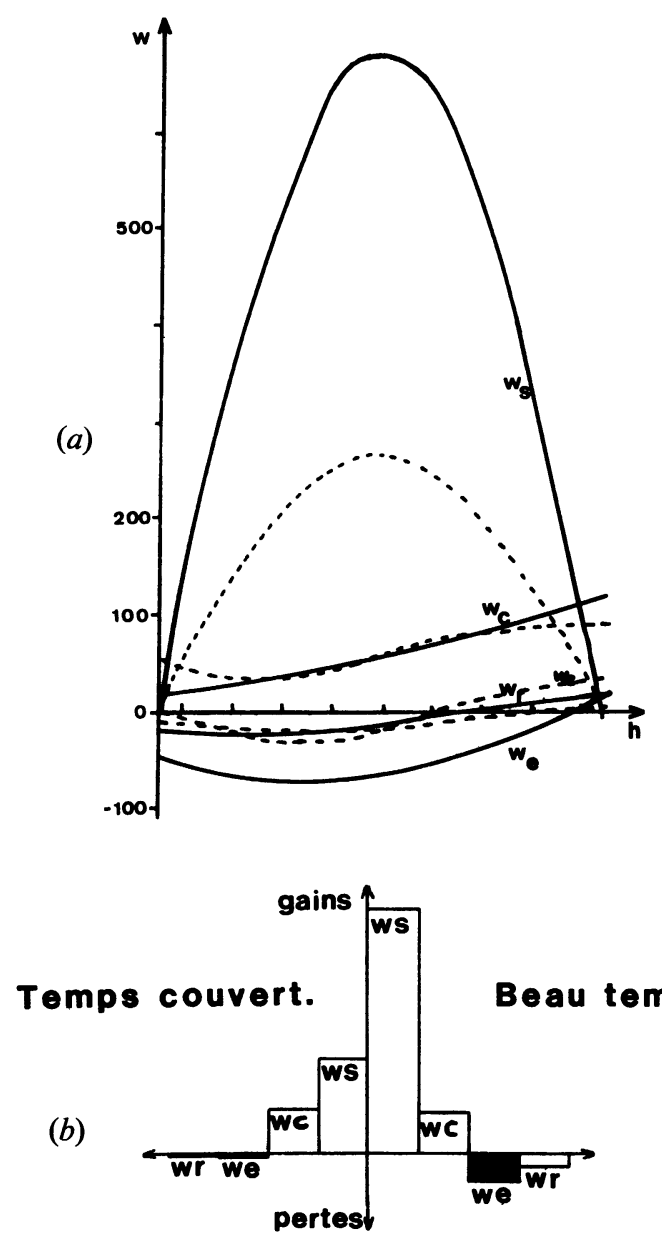

Fig. 4. - Comparaison entre les énergies convectives, évaporatives, par énergies de rayonnement et solaires pendant les heures d'ensoleillement (Nice - mars...) : représentation horaire $(a)$ et histogramme sur la saison de chauffe $(b)$.

[Comparison between energy collected by convection, evaporation, radiation with environment and solar energy during day hours (Nice - March) : horary diagram (a) and histogram for the heating period $(b)$.] solaires est difficile à obtenir. Ainsi les résultats de modélisation (Fig. 4) sont-ils décourageants pour ce qui concerne les apports par condensation.

Deux possibilités pour une amélioration du système s'en déduisent :

a) Faire circuler l'eau à l'intérieur d'un capteur sans vitrage : on gagne la réflexion à la surface du dioptre eau-air ( $3 \%$ d'énergie solaire en plus), sauf en présence de condensation-film, mais on perd un peu sur la convection et la condensation $\left(T_{\text {paroi }}>T_{\text {eau }}\right.$, les transferts calorifiques dûs à la condensation s'effectuent avec un rendement moindre); les problèmes de développement d'algues et d'évaporation sont évités.

- On peut circuler à débit plus grand l'eau n'étant plus contrainte à descendre le long du capteur par effet gravitationnel. L'écoulement peut être plus uniforme mais le capteur est plus onéreux. Par suite de l'échauffement de l'absorbeur surtout si celui-ci est constitué par un matériau comme le PVC, le béton ou le fibrociment, les pertes convectives sont plus importantes. Les gains par condensation sont maintenus et l'on n'est pas gêné par une utilisation en temps de pluie (trop plein d'eau à la sortie du capteur). En 1/2 saison, les températures d'eau en sortie de capteur sont suffisantes pour se passer de PAC.

Les efficacités de captage sont moindres mais les besoins en chauffage de l'habitation sont également réduits. Il y a économie de courant et l'on évite une surproduction de calories. Le COP annuel de l'installation s'en trouve amélioré.

La suppression de l'isolation arrière, double la surface d'échange pour les apports par convection et condensation; mais ceux-ci n'augmentent pas dans les mêmes proportions (débit d'air à l'arrière du capteur).

Une variante consiste à faire ruisseler l'eau sur la face arrière du capteur, le long d'un matériau poreux, où elle se maintient par tension superficielle (brevet Eternit).

b) Utiliser un liquide hygroscopique afin d'augmenter les apports par condensation. Un soin particulier doit être apporté aux tuyauteries à cause du caractère généralement corrosif de tels fluides. De même des précautions doivent être prises à cause des évaporations possibles de composés dangereux (évaporation d'HCl, 1 à $2 \%$ dans le cas d'une solution saline de $\mathrm{MgCl}_{2}$ ).

L'utilisation de solutions hygroscopiques est particulièrement intéressante : en remarquant que par mauvais temps il y a dilution de la solution tandis que par fort ensoleillement il y a concentration par évaporation, on réalise donc un stockage (qui peut être annuel) des apports par jours de beau temps pour les jours de mauvais temps. La conservation d'un tel stockage ne dépend pas de la température. Par ailleurs, le stockage peut être utilisé, hors capteur, pendant la nuit ou pendant les périodes de grands froids. 
Tableau III. - Energie récupérée à Nice par un capteur de $3 \mathrm{~m}$ de long pour une largeur unitaire. Température d'entrée de $6^{\circ}$ et un débit d'écoulement d'eau de $2,4 \mathrm{l} / \mathrm{min}$. Modélisation sur un hiver.

Beau temps

Temps couvert

\begin{tabular}{|c|c|c|c|c|}
\hline \multirow{2}{*}{ Novembre } & $11,27 \mathrm{kWh}$ & & $5,89 \mathrm{kWh}$ & $52 \%$ \\
\cline { 2 - 5 } & 0,84 & $57 \%$ & 1,13 & $60 \%$ \\
\hline \multirow{3}{*}{ Décembre } & $10,04 \mathrm{kWh}$ & & $4,45 \mathrm{kWh}$ & $44 \%$ \\
\cline { 2 - 5 } & 0,81 & $57 \%$ & 0,93 & $56 \%$ \\
\hline \multirow{2}{*}{ Janvier } & $11,17 \mathrm{kWh}$ & & $4,85 \mathrm{kWh}$ & $43 \%$ \\
\cline { 2 - 5 } & 0,78 & $57 \%$ & 0,88 & $56 \%$ \\
\hline \multirow{2}{*}{ Février } & $12,89 \mathrm{kWh}$ & & $5,76 \mathrm{kWh}$ & $45 \%$ \\
\cline { 2 - 5 } & 0,81 & $54 \%$ & 0,91 & $52 \%$ \\
\hline \multirow{2}{*}{ Mars } & $16,68 \mathrm{kWh}$ & & $7,72 \mathrm{kWh}$ & $46 \%$ \\
\cline { 2 - 5 } & 0,81 & $53 \%$ & 0,93 & $52 \%$ \\
\hline \multirow{2}{*}{ Avril } & $19,11 \mathrm{kWh}$ & & $11,59 \mathrm{kWh}$ & $61 \%$ \\
\cline { 2 - 5 } & 0,87 & $55 \%$ & 1,27 & $53 \%$ \\
\hline
\end{tabular}

Charge récupérée pendant la journée

\begin{tabular}{|l|l|}
\hline $5,89 \mathrm{kWh}$ & $52 \%$ \\
\hline 1,13 & $60 \%$ \\
\hline
\end{tabular}

pourcentage de l'énergie récupérée par temps couvert par rapport au beau temps

Rapport entre l'énergie récupérée l'après-midi et l'énergie totale récupérée pendant la journée

$$
\text { Eff. }=\frac{\text { Energie récupérée par jour }}{\text { Energie solaire incidente par jour }}
$$

3. Expérience de fonctionnement d'un capteur à ruissellement d'eau. - La figure 5 décrit l'appareil mis au point. Les mesures effectuées ont eu pour intérêt, non seulement de vérifier les performances d'un tel capteur, mais surtout d'approfondir la connaissance du phénomène d'évaporation-condensation.

Les différentes quantités mesurées ont été :

- le rayonnement solaire (pyranomètre Kipp and Zonen précision $5 \%$ ),

- la vitesse d'agitation de l'air au voisinage du film d'eau intégrée sur une durée donnée d'environ $1 \mathrm{~mm}$ (anémomètre Jules Richard),

- les températures d'air et d'eau à l'entrée et à la sortie du capteur (thermomètres à mercure de précision $0,2^{\circ} \mathrm{C}$ ),

- le débit par un compteur volumétrique (intégration sur une durée donnée d'environ $1 \mathrm{~mm}$ ), $5 \%$ ).
D'autres mesures plus ponctuelles ont été réalisées : températures le long du capteur, rayonnement réfléchi par le film d'eau (cellule photoélectrique).

Il est certain que les mesures effectuées l'ont été avec une précision qui aurait pu a priori être meilleure. Cependant des sources d'erreurs de provenances diverses existant par ailleurs, elles supprimaient toute justification à des mesures plus précises.

Ainsi :

- la surface exacte d'écoulement,

- la constance de l'épaisseur du film d'eau le long du capteur,

- l'agitation de l'air, variable d'un point à l'autre,

- la nécessité d'effectuer des mesures intégrées sur des données, d'environ 4 à 5 min (inertie du capteur, bacs de mesures...).

En définitive seules les énergies solaires incidente et totale récupérées étaient directement mesurées. $W_{\mathrm{c}}$ et $W_{\mathrm{R}}$ étaient calculées à partir des mesures; 


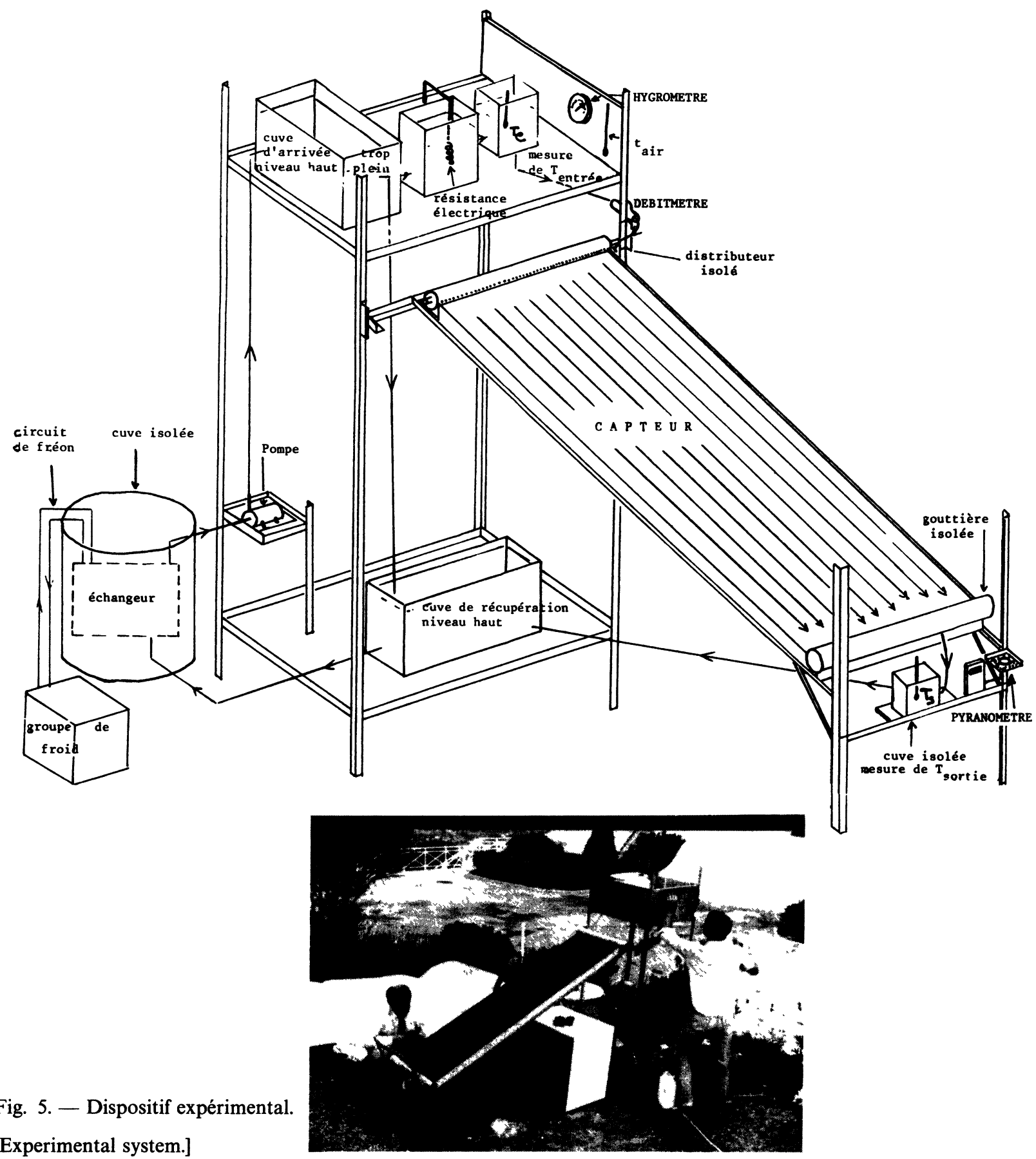

[Experimental system.]

$W_{\mathrm{E}}$ était d'une part déduite du reste, d'autre part calculée par les différentes relations étudiées.

Le fait de ne pouvoir mesurer $W_{\mathrm{E}}$ directement impliquait de rapporter les imprécisions portant sur les autres quantités. Le résultat en a été une distribution des points sur les figures sous forme de nuages ayant une dispersion notable.

La figure 6 est la meilleure description des résultats obtenus sur 8 jours de mesures. Les analyses des observations aboutissent aux conclusions suivantes :

Le phénomène de condensation n'est pas le phénomène inverse de celui de l'évaporation, surtout quand celle-ci se produit à une température comprise entre
$T_{\text {rosée }}$ et $T_{\text {air }}$; la loi de Dalton, tout comme les relations établies pour les déperditions de piscines ne peut donc s'appliquer aussi simplement.

Les diverses tentatives d'expression de $W_{\mathrm{c}}$ ou de $W_{\mathrm{E}}$ en fonction d'un quelconque paramètre n'ayant abouti qu'à la figuration d'un nuage de points très dispersé, il s'en suit, de par la figure 6 , que $W_{\mathrm{E}}$ et $W_{\mathrm{c}}$ sont inséparables l'un de l'autre. Lorsque la différence de pression partielle induit une évaporation alors que $T_{\text {rosée }}<T_{\text {eau }}<T_{\text {air }}$, tout se passe comme si une partie de l'énergie nécessaire à l'évaporation provenait de l'atmosphère.

Des mesures d'évaporation d'eau dans diverses 


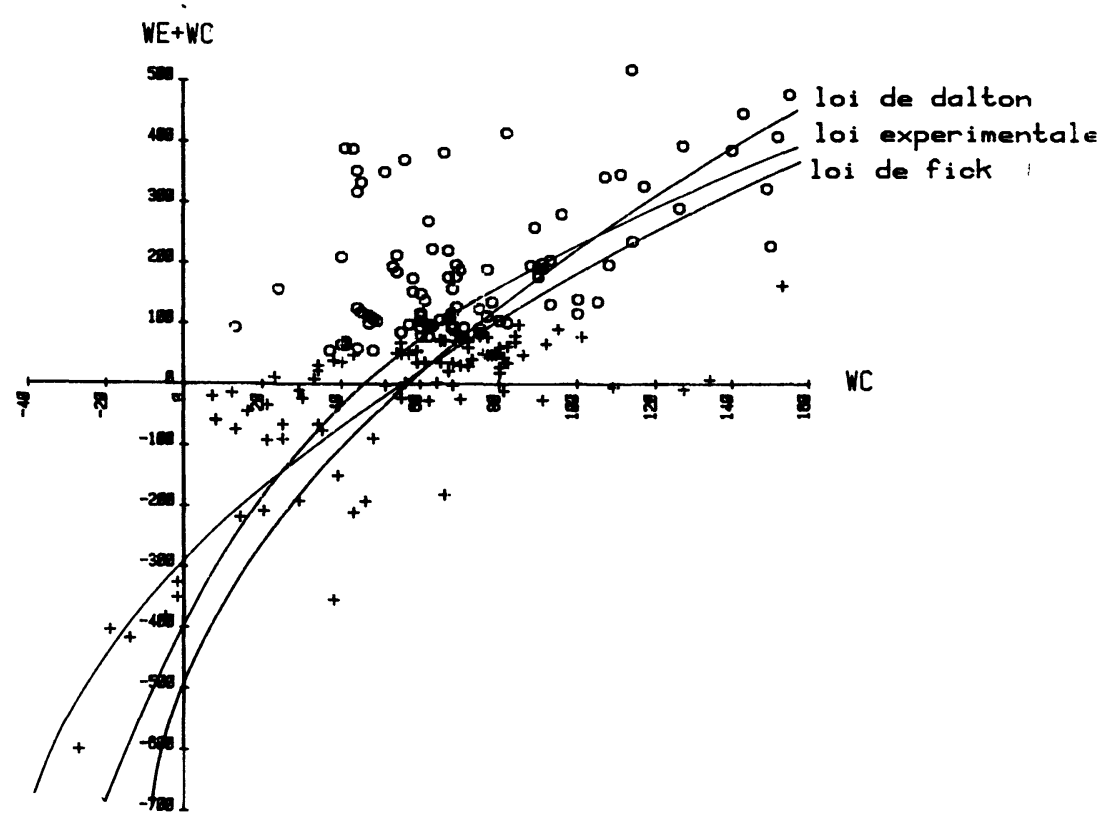

Fig. 6. - Energie échangée par déplacement de matière (évaporation + convection) en fonction de l'énergie convectée. $W_{\text {c }}$ est calculée et $W_{\mathrm{E}}$ déduite des autres mesures effectuées.

[Exchanged energy by displacement of matter (evaporation + convection) as a function of convected energy. $W$, is calculated, $W_{\mathrm{E}}$ is deducted from the others measures.]

conditions (en intérieur, en extérieur, avec un vent variable artificiel ou réel, avec ou non agitation de l'eau) ont mis en évidence l'importance du vent et surtout de l'agitation de l'eau. La tension superficielle au niveau de la couche limite eau-air a une grande influence; les effets de condensation sont renforcés.
La tension superficielle décroît avec la température suivant la loi (Réf. [5]) : $\sigma=\sigma_{0}(1+a \Delta t)$, avec

$$
\sigma_{250}=0,075 \mathrm{~N} / \mathrm{m} \text { et } a=-0,172(\mathrm{eau}) \text {. }
$$

L'épaisseur du film d'eau (0,1 mm en moyenne) a de

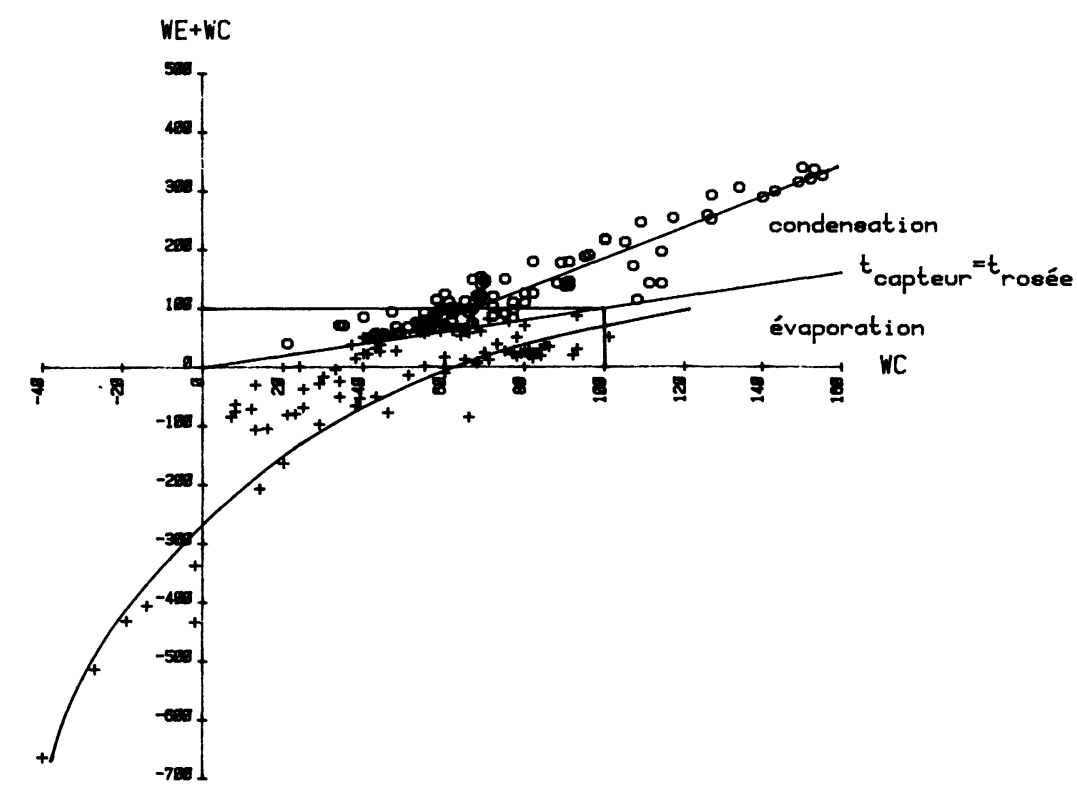

Fig. 7. - Energie échangée par mouvement de matière en fonction de l'énergie convective. $W_{\mathrm{E}}$ est obtenue par application de la loi de Fick aux mesures effectuées.

[Exchanged energy by displacement of matter as a function of convected energy. $W_{\mathrm{E}}$ is deducted from the Fick'slaw applied to the experimental measures.] 
plus son importance pour retenir l'eau au niveau du capteur (tension capillaire).

En définitive, les observations nous ont amené à modifier dans le programme l'expression utilisée pour calculer les échanges par évapo-condensation.

La chaleur de vaporisation de l'eau étant approximée, par une méthode des moindres carrés appliquée à une régression linéaire, par l'expression :

$$
L_{\mathrm{v}}=2498-2,413 t\left(t \text { en }{ }^{\circ} \mathrm{C}\right)\left(L_{\mathrm{v}} \text { en } \mathrm{J} / \mathrm{g}\right),
$$

(valable pour $0{ }^{\circ} \mathrm{C}<t<90^{\circ} \mathrm{C}$ ).

La relation choisie pour parfaire l'accord avec les observations est :

$$
W_{\mathrm{E}}=(17,39+0,017 t) \Delta p\left(1+\frac{V}{6}\right),
$$

où $\Delta p$ est exprimé en $\mathrm{mb}, t$ en ${ }^{\circ} \mathrm{C}$ et $V$ en $\mathrm{m} / \mathrm{s}$.

$\mathrm{Si}$ cette relation permet de décrire au mieux les observations, la loi de Fick donne néanmoins la meilleure cohérence interne (Fig. 7) entre les mesures. Ainsi cette relation est celle qu'il conviendrait d'adopter, au besoin en y ajustant les coefficients. Les imprécisions observées sont dues au fait que $W_{\mathrm{E}}$ est une déduction des autres observations : vitesse d'agitation de l'air, mesure du rayonnement solaire, des diverses températures, de la surface d'écoulement, du débit...

4. Couplage capteur à ruissellement-pompe à chaleur. - La nécessité de capter de l'énergie climatique impose des températures de circulation basses; il est donc nécessaire, en vue du chauffage d'habitation, de coupler le capteur à ruissellement à une P.A.C.

Cette dernière influe considérablement sur l'efficacité de captage réelle et sur les performances du système.

Afin de limiter les débits sur l'évaporateur (et par voie de conséquence la puissance des circulateurs), les P.A.C. eau/eau ont besoin d'une température de source

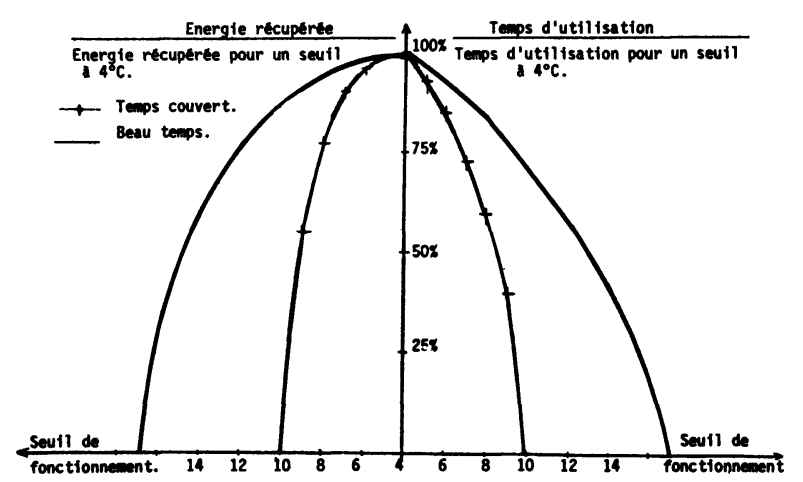

Fig. 8. - Temps d'utilisation du système pompe + capteur et énergie récupérable en fonction du seuil de fonctionnement de la pompe à chaleur (Toulouse - mars).

[Use time of the system, pump + collector, and collected energy as a function of the inlet temperature at the evaporator.] froide d'au moins $8^{\circ} \mathrm{C}$. Cela revient à se priver d'une partie des apports climatiques.

La figure 8 permet de visualiser l'évolution de l'énergie captée ainsi que le temps de fonctionnement en fonction du seuil de déclenchement de la P.A.C.

Du fait du couplage avec une P.A.C., les temps d'utilisation du système diminuent et les performances sont comparables à celles d'une P.A.C. air/air.

Sur ordinateur, le fonctionnement du système complet a été modélisé sur tout un hiver pour plusieurs villes de France (Fig. 9). Le taux maximum de couverture des besoins pour une maison témoin a été calculé en différentes régions et une relation linéaire empirique en a été déduite :

$$
\begin{array}{lll}
c=0,77 \sigma+0,048 t_{\mathrm{r}} & \text { (pour un COP égal à 3) } \\
c=1,1 \quad \sigma+0,033 t_{\mathrm{r}} & \text { (pour un COP égal à 4) }
\end{array}
$$

avec

$\sigma:$ fraction d'insolation moyenne des mois d'octobre à avril pour le site considéré,

$t_{\mathrm{r}}$ : moyenne arithmétique des températures de rosée pour la même période.

Le coefficient $c$ représente les besoins maximaux que peut satisfaire le capteur à ruissellement, compte non tenu de l'énergie fournie par le compresseur de la P.A.C.

La maison témoin déplacée d'un endroit à l'autre de la France a les caractéristiques suivantes : volume $300 \mathrm{~m}^{3}$. $G=1 \mathrm{~W} / \mathrm{m}^{3}$. ${ }^{\circ} \mathrm{C}$. Ruissellement en surface de toiture sud $60 \mathrm{~m}^{2}$. Surface de vitrage sud : $7 \mathrm{~m}^{2}$.

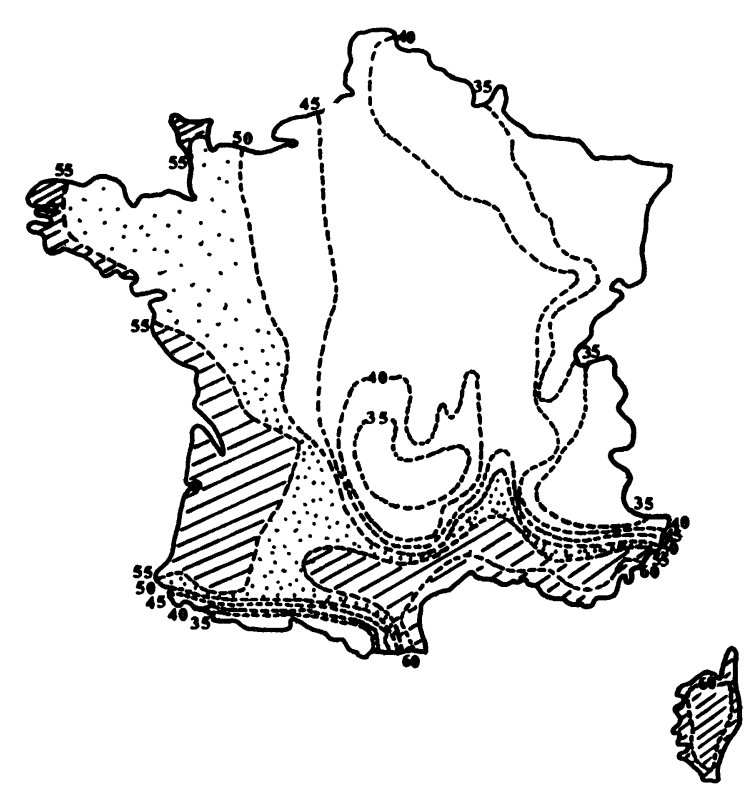

Fig. 9. - Zones d'utilisation du capteur à ruissellement d'eau. Pourcentage des besoins couverts par le système (cas de la maison témoin).

[Geographical zones of application for the system pump + cold streaming collector. Percentage of the covered heating uses in the case of the model house.] 
P.A.C. eau/eau de type GT 90 (Leroy-Somer) utilisée entre une source froide à $8^{\circ} \mathrm{C}$ et un plancher chauffant à $30^{\circ} \mathrm{C}(\mathrm{COP}=3)$. Il est certain qu'un bâtiment avec d'autres caractéristiques et un autre type de pompe peut avoir d'autres performances.

La zone d'utilisation du système capteur à ruissellement + P.A.C. regroupe évidemment les côtes atlantique et méditerranéenne qui sont les plus douces en température et les plus humides (Fig. 9). Dans ces régions, le couplage des deux systèmes permet une couverture des besoins 5 à $10 \%$ supérieure à celle obtenue avec une P.A.C. seule. Les courbes de la figure 9 sont issues des études effectuées pour 41 stations régulièrement distribuées sur toute la France.

Pour augmenter les performances du système (taux de couverture des besoins) il faut pouvoir se passer de la P.A.C. en demi-saison par ciel dégagé ; sans cela le COP global annuel est limité par celui de la pompe à chaleur.

A remarquer que l'asservissement du débit aux conditions climatiques permet de couvrir environ $5 \%$ de besoins supplémentaires, mais que la complexité augmente et que le compresseur de la P.A.C. doit être à régime variable (P.A.C. à moteur thermique).

En conclusion, le ruissellement d'eau à l'air libre ne présente pas un intérêt décisif : en effet, partout où ce système est applicable, il entre en concurrence avec une pompe à chaleur air/air ou air/eau (régions douces et humides où l'enthalpie de l'air est élevée). Pour étendre les possibilités du système, il convient d'en améliorer les performances :

- en circulant dans un radiateur plastique fin non isolé sur sa face inférieure,

- en circulant avec un fréon à très basse température les apports par condensation et convection sont alors notablement augmentés (Réf. [13]), mais d'autres problèmes apparaissent,

- en circulant avec de l'eau glycolée dont la température de fusion serait de l'ordre de $-20^{\circ} \mathrm{C}$,

- en utilisant des solutions hygroscopiques. De façon à condenser l'humidité de l'air, il faut circuler en surface mais se pose alors le problème des jours de pluie (dilution). Ceci amène à transformer un captage surfacique en captage volumique : les saumures reposent dans des bacs empilés dans un abri fortement ventilé, elles s'échauffent par convection et condensation et échangent leur chaleur avec le circuit d'eau froide de la P.A.C.

Les solutions hygroscopiques présentent par ailleurs l'intérêt de constituer des stockages indépendants de la température.

5. Utilisation de sels hygroscopiques. - L'utilisation de solutions salines permet un abaissement de la pression partielle de vapeur saturante. D'où un fonctionnement possible à très basse température sans risque de gel.

Pour situer l'intérêt de telles solutions, il suffit de savoir qu'une saumure qui permettrait la condensation d'1/2 litre d'eau par heure et par $\mathrm{m}^{2}$ de capteur fournirait une puissance de $335 \mathrm{~W} / \mathrm{m}^{2}$ pour les seuls apports par condensation. Une maison, comme celle citée plus haut, n'aurait besoin que de $10 \mathrm{~m}^{2}$ de capteurs et d'un stockage de $5 \mathrm{~m}^{3}$, stockage qui serait alors intersaisonnier.

Parmi les sels les plus hygroscopiques, citons le chlorure de magnésium $\left(\mathrm{MgCl}_{2}\right)$ qui a fait l'objet d'expériences avec le capteur décrit ci-dessus. Par la suite, il pourrait être remplacé par du $\mathrm{LiCl}$ plus hygroscopique, moins corrosif (monochlorure), mais aussi plus cher à l'achat. Certains glycols encore plus hygroscopiques que le chlorure de lithium, peu chers et non corrosifs, seraient finalement les meilleurs produits à retenir.

Les sels hydratés ont une chaleur spécifique $C_{\mathrm{p}}$ et une masse volumique $\rho$ variable en fonction de la concentration. La figure 10 décrit la variation du produit $\left(\rho C_{\mathrm{p}}\right)$ en fonction de la concentration pour une solution de chlorure de magnésium. Il est important que la valeur de ce produit reste aussi élevée que possible de façon à limiter le débit (pertes de charges) et les élévations de températures. Par ailleurs, des concentrations élevées ne sont pas possibles sans l'apparition de cristaux solides (à $20^{\circ} \mathrm{C}$ on ne peut dépasser $C=0,54$, soit dissoudre plus de $54,25 \mathrm{~g}$ de $\mathrm{MgCl}_{2}$ dans $100 \mathrm{~g}$ d'eau). La présence de cristaux n'est pas un inconvénient : la sursaturation (présence simultanée des phases solide et liquide) représente un stockage qui peut être renouvelé en début ou en cours d'hiver (adjonction de sel). Elle assure un maintien de la concentration pendant les heures de fonctionnement par jours de'mauvais temps.

68 mesures étalées sur 3 jours ont été effectuées (Fig. 11). Elles montrent un relèvement moyen du point de condensation de $5,3^{\circ}$ pour une solution à $10 \%$ de concentration en $\mathrm{MgCl}_{2}$; l'expression déduite pour la pression partielle de vapeur saturante (dans la solution concentrée à $10 \%$ de $\mathrm{MgCl}_{2}$ ) est :

$$
p=10^{\left(0,187+0,03 t_{\text {sol. }}^{0}\right)} \text {. }
$$

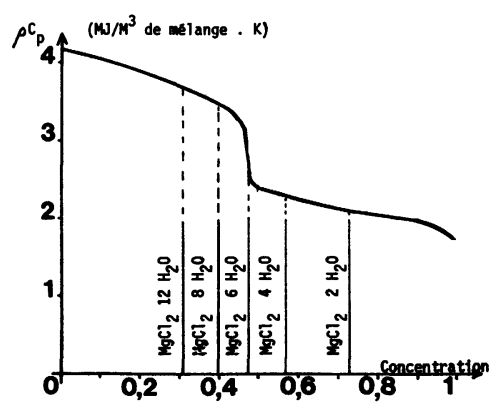

Fig. 10. - Variation du produit $\rho C_{p}$ en fonction de la concentration en chlorure de magnésium.

$\left[\rho C_{p}\right.$ value as a function of the concentration $\left.\left(\mathrm{MgCl}_{2}\right) \cdot\right]$ 


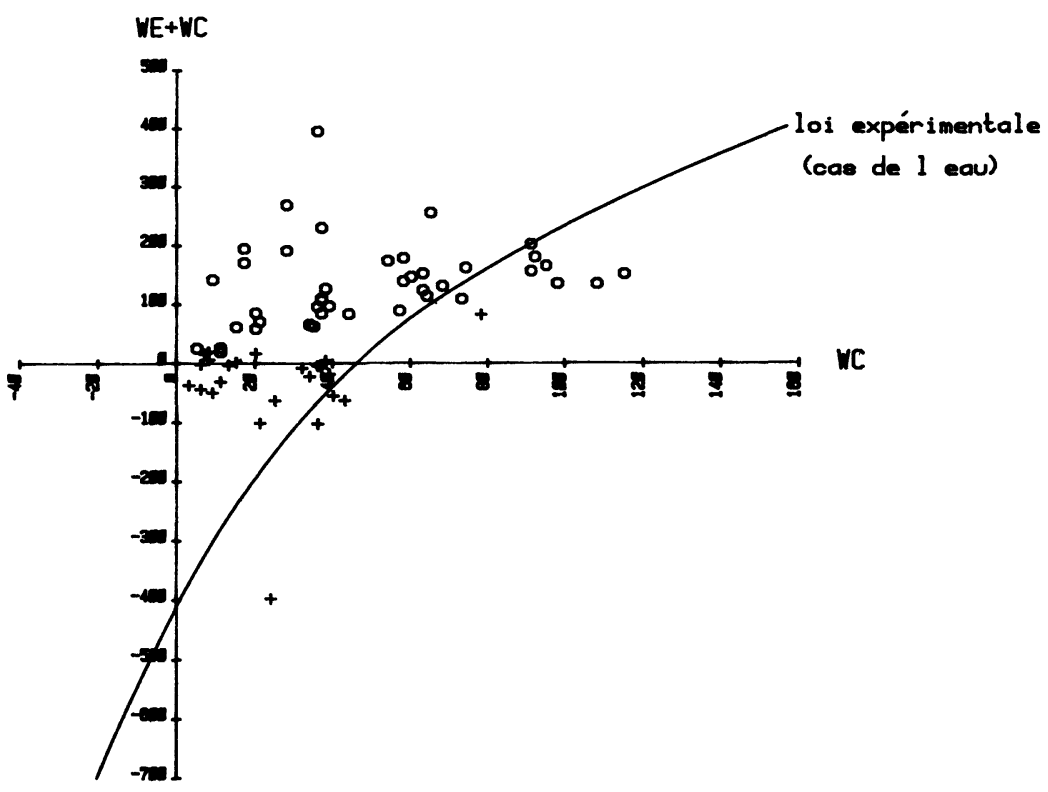

Fig. 11. - Energie échangée par mouvement de matière en fonction de l'énergie échangée convectivement. Cas d'une saumure de $\mathrm{MgCl}_{2}$ de concentration 3 à $15 \%$.

[Exchanged energy by displacement of matter as a function of convected energy $\left(\mathrm{MgCl}_{2}\right.$ solution concentrated about $\left.10 \%\right)$.]

Le relèvement du point de condensation diminue jusqu'à supprimer les effets contradictoires de convection et d'évaporation qui existent lorsque la température du fluide est comprise entre la température de rosée et celle de l'air. La figure 12 montre pour un sel particulier $(\mathrm{LiCl})$ l'équivalence entre concentration et pression de vapeur saturante. Il s'ensuit un renforcement des apports convectifs, l'air étant "encouragé dans son mouvement " par l'attrait hygroscopique de la solution. C'est pourquoi ces deux effets, condensatifs et convectifs ne peuvent être dissociés.

6. Conclusion. - Le capteur à ruissellement d'eau ne présente en lui-même qu'un intérêt pédagogique.

L'utilisation de saumures est la solution à rechercher, mais sous une forme de capteur volumique (brumisation dans une tour de refroidissement par exemple) et non pas surfacique, en raison des problèmes posés par l'écoulement de telles solutions. Les apports solaires seraient alors obtenus par des capteurs vitrés classiques ou des capteurs de piscine, ce qui améliorerait l'efficacité du système par beau temps (apports et concentration de la solution). La ventilation forcée au sein de la solution augmenterait quant à elle les échanges par évaporation-condensation. Une telle solution, pour un groupe d'habitations, pourrait très bien approcher l'optimum financier qui est à rechercher pour une installation de chauffage. Une telle étude a d'ailleurs été entreprise (Réf. [14]) et sera présentée ultérieurement.

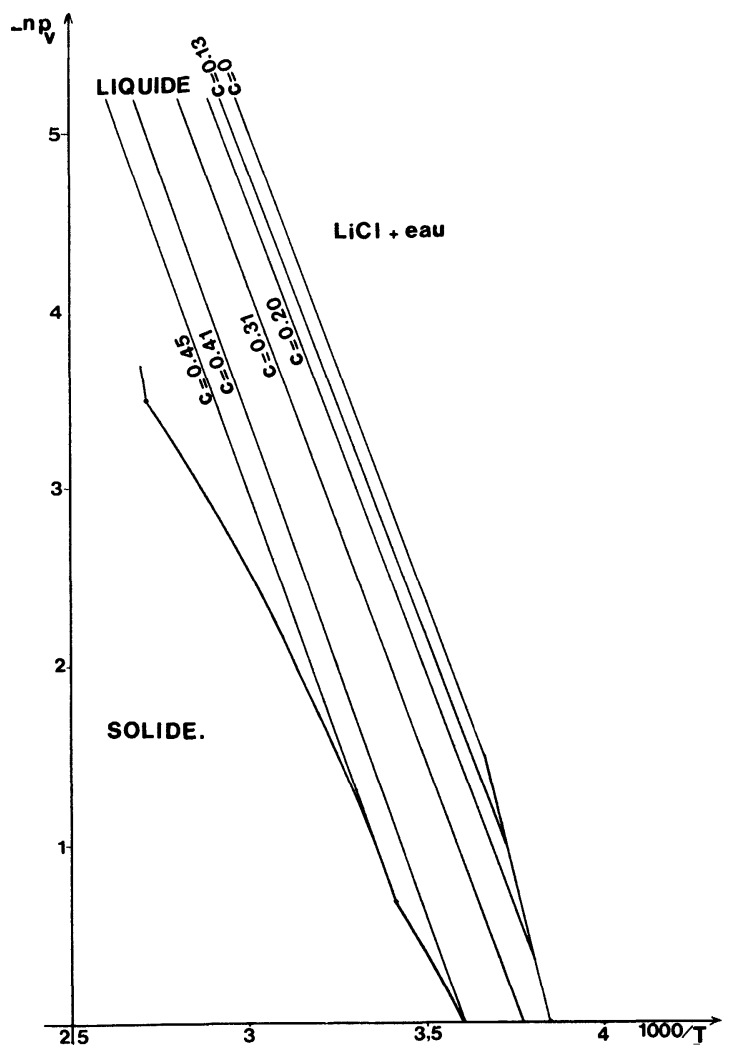

Fig. 12. - Diagramme de phase $\mathrm{LiCl}+\mathrm{H}_{2} \mathrm{O}$. (Communication personnelle de J. Bathiebo et J.-D. Sylvain, CNRS/ ETS.)

[Phase diagram of $\mathrm{LiCl}$.] 


\section{Bibliographie}

[1] BERgER, X., Chauffage solaire et stratégies de chauffage. Revue Phys. Appl. 16 (1981) 225.

[2] GARnier, F., Simulateur de météo pour St-Quentin. Détermination de la température de ciel (Rapport de stage ENSIMEV) 1982.

[3] McAdams, Heat transmission (McGraw Hill) 1954.

[4] Rapport C.S.T.B., Hygrothermique des piscines.

[5] Manuel des Techniques de l'Ingénieur (Dunod) Tome II 1961, p. 218

[6] Tusseau, P., Gaz humides (Techniques de l'Ingénieur, p. 600).

[7] Delvaux, L., Contribution à l'étude de l'évaporation par convection forcée turbulente à partir d'une surface plane horizontale (Thèse Paris) 1967.

[8] Pelletret, R., Association de capteurs sans vitrage et de pompe à chaleur. Capteur à condensation (Rapport de fin d'études ENSIMEV) 1981.
[9] RAY, P., Capteur à ruissellement (Rapport de fin d'études Ecole Centrale de Lyon) 1980.

[10] Berger, X., Simulateur de météorologie pour habitat solaire - Région niçoise (C.N.R.S.) 1982.

[11] Berger, X., Capteur à condensation pour une maison à Juan-les-Pins (Rapport interne C.N.R.S. Groupe Ecothermique Solaire) 1980.

[12] SCHNEIDER, M., Capteur à ruissellement (Concours COMES et rapport Plan Construction Maison Energie Zéro) 1979.

[13] CoRdier, A. et Gessinn, G., Chauffage solaire utilisant une pompe à chaleur et des capteurs froids. (Rév. Int. d'Héliotechnique. COMPLES $2 \mathrm{e}$ semestre) 1978.

[14] Maschi, M. et Riv, R., Récupération de chaleur sur l'air par une tour de refroidissement (Stage de fin d'études Ecole des Mines) 1982. 